\title{
Impacto del COVID-19 en el empleo en la ciudad de Quito
}

\author{
Fecha de recepción: 15-02-2021 • Fecha de aceptación: 05-04-2021 • Fecha de publicación: 10-06-2021
}

\author{
Michel Esthefanía Arévalo Ávila' \\ Puntonet S.A., Ecuador \\ esthefania.arevalo.avila@gmail.com \\ https://orcid.org/0000-0001-7652-6509 \\ Jhonnatan Vinicio Reascos Tapia ${ }^{2}$ \\ Puntonet S.A., Ecuador \\ jhovi_reta_14@hotmail.com \\ https://orcid.org/0000-0001-7750-7641 \\ Héctor Sebastián Pérez Manosalvas ${ }^{3}$ \\ Universidad Tecnológica Israel, Ecuador \\ hperez@uisrael.edu.ec \\ https://orcid.org/0000-0001-5187-1015
}

\section{RESUMEN}

El artículo presentado entendió a la pandemia como un problema que potencialmente podía estar afectando al empleo en términos de despidos y desmejoramiento de las condiciones de trabajo. El objetivo es determinar el impacto del COVID-19 en el empleo en la ciudad de Quito, Ecuador. Se implementó una metodología cuantitativa y empírica-descriptiva, se recolectaron datos de 50 personas de la ciudad de Quito para evaluar el impacto de la pandemia en los puntos señalados. Los resultados mostraron que las mujeres eran las más afectadas por la pérdida de empleos, mientras que ambos sexos estaban siendo afectados por un desmejoramiento de sus condiciones laborales, donde el estrés y la sobrecarga de trabajo eran comunes. La solución, según se evaluó, pasa por la aplicación de estrategias de las empresas públicas y privadas en términos del manejo de su talento humano. 
PALABRAS CLAVE: empleo, Covid-19, pandemia, desempleo, despedidos.

\section{ABSTRACT}

The article presented understood the pandemic as a problem that could potentially be affecting employment in terms of layoffs and deterioration of working conditions. The objective is to determine the impact of COVID-19 on employment in the city of Quito, Ecuador. A quantitative and empirical-descriptive methodology was implemented, data were collected from 50 people in the city of Quito to evaluate the impact of the pandemic on the points indicated. The results showed that women were the most affected by the loss of jobs, while both sexes were being affected by a deterioration of their working conditions, where stress and work overload were common. The solution, as assessed, lies in the implementation of strategies by public and private companies in terms of managing their human talent.

KEYWORDS: employment, Covid-19, pandemic, unemployment, dismissal. 


\section{Introducción}

El empleo es uno de los motores principales del desarrollo económico y social para cualquier país (Kubicek \& Korunka, 2017). Gracias al empleo, no sólo es posible aumentar la competitividad productiva de las empresas, sino también desarrollar una población con mayores oportunidades de compra, lo cual ayuda que las condiciones de vida de esa población sean cada vez mejores (Young Lee \& Lee, 2018). Por esta razón, y otras más, el empleo es un bien primordial que muchas naciones protegen a toda costa.

El problema radica en que el empleo, por cuestiones de economía básica, suele ser muy afectado en tiempos de crisis; tiempos en los que la producción decae drásticamente y es imposible mantener una masa trabajadora amplia en cada empresa (González, 2017). Estas situaciones, a veces previsibles, a veces no, suelen impactar negativamente en el empleo, bien sea a través de la reducción de los salarios (y en ocasiones las condiciones laborales) o bien reduciéndose la cantidad de trabajadores por empresa. Estas realidades han acontecido en todo el mundo a través del tiempo, repitiéndose nuevamente en este año 2020 debido a la pandemia por COVID-19 (Arce, 2020).

La pandemia denominada COVID-19 que apareció a finales de diciembre del 2019 en Wuhan, China, ha causado muchos problemas a nivel económico en todo el mundo, donde Ecuador ha sido uno de los países más afectados de la región de América Latina.

Según el Banco Central del Ecuador (2020), el PIB del país decreció la pasmosa cifra de 12,4\% sólo en el segundo trimestre del 2020 , situación sólo comparable con la de la crisis previa a la dolarización. La causa principal de este impresionante declive de la economía nacional fue, sin duda, el COVID-19 (Quevedo Barros, Vásquez Lafebre, Quevedo Vasquez, \& Pinzón Prado, 2020). Por la facilidad de transmisión del virus, la población se vio obligada a un tiempo de confinamiento, lo cual trajo consigo un declive en la economía nacional, sobre todo en el sector comercial y turístico.

El confinamiento provocó una reducción drástica de las ventas en casi todos los sectores económicos del país y, con ello, una pérdida masiva de empleos. Esto, según se comprende, sucedió e incluso sigue sucediendo en todas las provincias y cantones del país (Cevallos, Calle, \& Ponce, 2020). Pero, pese a esta generalidad, surge la duda específica de conocer cómo impactó el COVID-19 en el empleo de una ciudad en particular, una de las más importantes para el país: Quito. En este sentido, el presente artículo se enfrasca en, justamente, dar a conocer esta realidad en el cantón, para así ofrecer una perspectiva más completa de hasta qué punto la pandemia afectó a la ciudad en términos de empleo y la perspectiva a futuro acerca del mismo.

Dicho planteamiento investigativo se justifica por ser una manera de arrojar luz sobre una realidad que todavía no ha acabado. La pandemia aún está lejos de terminar y es necesario evaluar correctamente, desde cualquier trinchera, los impactos de la misma sobre diferentes dimensiones, donde el empleo es una de las más importantes. En este sentido, este artículo resulta muy importante como herramienta contextual sobre el empleo en la ciudad de Quito a raíz de la pandemia. También resultará útil para referenciar y servir de base a otros trabajos futuros que 
podrán encontrar en este artículo un exponente bastante completo del impacto del COVID-19 en el empleo en la ciudad de Quito. También se justifica desde lo académico, al ser este un trabajo capaz de permitir el análisis crítico de la situación desde las bases obtenidas por quien suscribe con relación a la profesión que se cursa.

De esta manera, se establece como objetivo general determinar el impacto del COVID-19 en el empleo en la ciudad de Quito. Como objetivos específicos, se determinan tres, que permitirán alcanzar el objetivo general planteado. Dicho esto, los específicos serían: analizar las consecuencias que tuvo el COVID-19 en la pérdida de empleos en la ciudad de Quito; evaluar el impacto que tuvo el COVID-19 en la degradación de las condiciones de empleo en la ciudad de Quito; valorar las mejores opciones que poseen las empresas y el sector público para reducir los impactos causados por COVID-19 en el empleo en la ciudad de Quito.

\section{Metodología}

Este artículo aplicó una metodología de enfoque cualitativo y cuantitativo. López Roldán y Fachelli (2015) aseguran que las investigaciones de tipo cuantitativa son ideales dentro de las ciencias sociales, ofreciendo ventajas de generalización de resultados y descripción rápida de las variables de estudio. También es un enfoque metodológico confiable, ya que permite la aplicación de técnicas e instrumentos cuantitativos que son los adecuados cuando se desea realizar análisis estadísticos de una realidad en concreto; por otro lado, Sampieri (2003) menciona que la metodología de enfoque cualitativa utiliza recolección de datos sin medición numérica para descubrir o afinar preguntas de investigación y puede o no probar hipótesis en su proceso de interpretación.

Con respecto al tipo de estudio, el mismo se consideró empírico y descriptivo. El nivel de conocimiento empírico, según Martínez (2017) son aquellos que permiten aplicar conocimientos y evaluar una realidad en el campo, es decir, en donde ocurre en ese momento u ocurrió el fenómeno en cuestión. Como la pandemia sigue su curso y el efecto en Quito sigue siendo afectado por ella, se evidenció la pertinencia de su aplicación para este trabajo.

Finalmente, que el trabajo se haya hecho de tipo descriptivo pasó por el hecho y la necesidad de requerirse información de ciertas variables para conocer el impacto del COVID-1en el empleo. La descripción sería en este caso otorgada por las consideraciones propias de las personas a abordar a través de los instrumentos de investigación.

Sobre la población y muestra, se consideró como población a todas las personas laboralmente capaces en la ciudad de Quito, mientras que la muestra fue seleccionada por conveniencia, a través del muestreo por bola de nieve. Con dicha técnica, se logró abordar a un total de 50 personas de la ciudad de Quito. Como instrumento de recolección de datos, la técnica que se utilizó fue el cuestionario debido a la conveniencia aplicativa y la versatilidad para el análisis de resultados que, por otro lado, se realizó mediante estadística descriptiva.

El cuestionario es de autoría de los investigadores y consta de 19 pregunta, mismas que permitirán conocer a fondo la situación laboral actual en la ciudad de Quito. 
Es importante mencionar que para el desarrollo de esta investigación se trató de aplicar una fórmula de muestreo finito; sin embargo, debido a factores sociales, problemas ocasionados por la pandemia y sobre todo el tiempo limitado para su desarrollo, se optó por el muestreo probabilístico, por conveniencia a través de bola de nieve.

\section{Resultados}

De las 50 personas evaluadas, los resultados de la aplicación de la encuesta se muestran a continuación. La Tabla 1 propone las preguntas preliminares. Como se puede observar, de las 50 personas evaluadas 40 poseían edades entre los 18 a 30 años, mientras que 10 tenían una edad comprendida entre los 30 y 50 años. En total, 31 eran hombres y 19 mujeres, 28 trabajaban en el sector público y 22 en el sector privado, perdiendo 34 personas sus puestos de trabajo, con tan sólo 16 conservándolo durante la pandemia.

Tabla 1.

Resultados de las preguntas preliminares.

\begin{tabular}{|l|l|}
\hline Pregunta & Respuestas \\
\hline Edad & $\begin{array}{l}18 \text { a } 30 \text { años: } 40 \text { personas } \\
30 \text { a } 50 \text { años: } 10 \text { personas. }\end{array}$ \\
\hline Sexo & $\begin{array}{l}\text { Hombres: } 31 \text { personas. } \\
\text { Mujeres: } 19 \text { personas. }\end{array}$ \\
\hline Sector & $\begin{array}{l}\text { Público: } 28 \text { personas. } \\
\text { Privado: } 22 \text { personas. }\end{array}$ \\
\hline ¿Perdió su empleo? & $\begin{array}{l}\text { Perdió su puesto de trabajo: 34 personas. } \\
\text { No perdió su puesto de trabajo: 16 personas }\end{array}$ \\
\hline
\end{tabular}

Fuente: elaboración propia

En las Tablas 2, 3, 4 y 5 se ofrecen los resultados del cuestionario para los hombres y mujeres encuestados. Debajo de cada una de estas tablas se ofrece el análisis correspondiente para las mismas. Cabe resaltar que se empleó sólo estadística descriptiva para la generación de las tablas. 
Tabla 2.

Resultados de las mujeres que no perdieron su empleo.

\begin{tabular}{|l|l|l|l|l|}
\hline \multicolumn{2}{|c|}{ Mujeres que no perdieron su empleo: 1 } \\
\hline Sector privado: & 0 & \multicolumn{2}{l|}{$0,00 \%$} \\
\hline Sector público: & 1 & \multicolumn{2}{l|}{$3,50 \%$} \\
\hline$\#$ & Pregunta & Si & \multicolumn{1}{l}{ No } \\
\hline 1 & $\begin{array}{l}\text { Desde la pandemia, tengo más trabajo que antes, pero no me aumentaron el sueldo por } \\
\text { ello. }\end{array}$ & $100 \%$ & $0 \%$ \\
\hline 2 & Sufrí una reducción de mi sueldo o de beneficios que percibía antes de la pandemia. & $0 \%$ & $100 \%$ \\
\hline 3 & Algunos de mis compañeros de trabajo perdieron sus empleos a causas de la pandemia. & $100 \%$ & $0 \%$ \\
\hline 4 & Algunos de mis familiares perdieron sus puestos de trabajo a causas de la pandemia. & $100 \%$ & $0 \%$ \\
\hline 5 & El trabajo se ha vuelto más complicado, más estresante y menos satisfactorio. & $100 \%$ & $0 \%$ \\
\hline 6 & Para no perder mi empleo, dejé de percibir salario durante varios meses... & $0 \%$ & $100 \%$ \\
\hline 7 & Actualmente trabajo mediante teletrabajo. & $0 \%$ & $100 \%$ \\
\hline 8 & La empresa me ha suministrado los equipos que requiero para poder trabajar desde casa. & $0 \%$ & $100 \%$ \\
\hline
\end{tabular}

Fuente: elaboración propia

De acuerdo con la Tabla 2, apenas una de las mujeres consultadas no perdió su empleo, esta mujer trabajaba en el sector público, e indicó que ha aumentado su carga laboral en la pandemia. También, a su alrededor, compañeros de trabajo y familiares se han visto afectados por la situación de la pandemia a nivel laboral.

Tabla 3.

Resultados de las mujeres que perdieron su empleo.

\begin{tabular}{|c|c|c|c|c|}
\hline \multicolumn{5}{|c|}{ Mujeres que perdieron su empleo: 18} \\
\hline \multicolumn{2}{|c|}{$\begin{array}{l}\text { Sector } \\
\text { privado: }\end{array}$} & 10 & \multicolumn{2}{|l|}{$45,45 \%$} \\
\hline \multicolumn{2}{|c|}{ Sector público: } & 8 & \multicolumn{2}{|l|}{$28,57 \%$} \\
\hline \# & \multicolumn{2}{|c|}{ Pregunta } & Si & No \\
\hline 1 & \multicolumn{2}{|c|}{ Al ser despedido, me pagaron los beneficios de ley según correspondía. } & $44,44 \%$ & $55,56 \%$ \\
\hline 2 & \multicolumn{2}{|c|}{ Hubo otros compañeros en la empresa que perdieron sus empleos en la pandemia. } & $66,67 \%$ & $33,33 \%$ \\
\hline 3 & \multicolumn{2}{|c|}{ En mi familia hubo personas que perdieron sus empleos durante la pandemia. } & $66,67 \%$ & $33,33 \%$ \\
\hline 4 & \multicolumn{2}{|c|}{ La empresa que me despidió se declaró en quiebra. } & $22,22 \%$ & $77,78 \%$ \\
\hline 5 & \multicolumn{2}{|c|}{ Actualmente ya poseo otro empleo. } & $5,56 \%$ & $94,44 \%$ \\
\hline 6 & \multicolumn{2}{|c|}{ La empresa nos ofreció posibilidad de reenganche en un futuro... } & $0,00 \%$ & $100 \%$ \\
\hline 7 & \multicolumn{2}{|c|}{ Si no me hubiesen despedido, igualmente hubiese renunciado poco después. } & $0,00 \%$ & $100 \%$ \\
\hline
\end{tabular}

Fuente: elaboración propia

Un total de 18 mujeres (Tabla 3) perdieron sus puestos de trabajo, representando el 36\% de la muestra evaluada. Estas mujeres expresaron en su mayoría que no les pagaron los beneficios correspondientes, no le ofrecieron reenganche futuro, pese a que la empresa no quebró. La mayoría manifestó que, en su entorno, ya sean familiares o amigos, perdieron su empleo a causas de la pandemia. 
Tabla 4.

Resultados de los hombres que no perdieron su empleo.

\begin{tabular}{|c|c|c|c|c|}
\hline \multicolumn{5}{|c|}{ Hombres que no perdieron su empleo: 15} \\
\hline \multicolumn{2}{|c|}{ Sector privado: } & 7 & \multicolumn{2}{|l|}{$31,82 \%$} \\
\hline \multicolumn{2}{|c|}{ Sector público: } & 8 & \multicolumn{2}{|l|}{$28,57 \%$} \\
\hline$\#$ & \multicolumn{2}{|c|}{ Pregunta } & Si & No \\
\hline 1 & \multicolumn{2}{|c|}{ Desde la pandemia, tengo más trabajo, pero no me aumentaron el sueldo por ello. } & $100 \%$ & $0 \%$ \\
\hline 2 & \multicolumn{2}{|c|}{$\begin{array}{l}\text { Sufrí una reducción de mi sueldo o de beneficios que percibía antes de la } \\
\text { pandemia. }\end{array}$} & $20 \%$ & $80 \%$ \\
\hline 3 & \multicolumn{2}{|c|}{$\begin{array}{l}\text { Algunos de mis compañeros de trabajo perdieron sus empleos a causas de la } \\
\text { pandemia. }\end{array}$} & $86,67 \%$ & $13,33 \%$ \\
\hline 4 & \multicolumn{2}{|c|}{$\begin{array}{l}\text { Algunos de mis familiares perdieron sus puestos de trabajo a causas de la } \\
\text { pandemia. }\end{array}$} & $100 \%$ & $0 \%$ \\
\hline 5 & \multicolumn{2}{|c|}{ El trabajo se ha vuelto más complicado, más estresante y menos satisfactorio. } & $90 \%$ & $10 \%$ \\
\hline 6 & \multicolumn{2}{|c|}{ Para no perder mi empleo, dejé de percibir salario durante varios meses... } & $0 \%$ & $100 \%$ \\
\hline 7 & \multicolumn{2}{|c|}{ Actualmente trabajo mediante teletrabajo. } & $40 \%$ & $60 \%$ \\
\hline 8 & \multicolumn{2}{|c|}{$\begin{array}{l}\text { La empresa me ha suministrado los equipos que requiero para poder trabajar } \\
\text { desde casa. }\end{array}$} & $0 \%$ & $100 \%$ \\
\hline
\end{tabular}

Fuente: elaboración propia

En relación a los hombres (Tabla 4) que no perdieron sus puestos de trabajo, estos fueron 15 en total (30\% de la muestra). No obstante, la mayoría indicó que, por la pandemia, tenía más trabajo que antes con el mismo sueldo, no sufrió una degradación de los beneficios que recibía, familiares y amigos perdieron su puesto de trabajo y se ha vuelto su empleo más estresante que en el pasado.

Tabla 5.

Resultados de los hombres que perdieron su empleo.

\begin{tabular}{|c|c|c|c|c|}
\hline \multicolumn{5}{|c|}{ Hombres que perdieron su empleo: 16} \\
\hline \multicolumn{2}{|c|}{ Sector privado: } & 5 & \multicolumn{2}{|l|}{$22,73 \%$} \\
\hline \multicolumn{2}{|c|}{ Sector público: } & 11 & \multicolumn{2}{|l|}{$39,29 \%$} \\
\hline \# & \multicolumn{2}{|l|}{ Pregunta } & Si & No \\
\hline 1 & \multicolumn{2}{|c|}{ Al ser despedido, me pagaron los beneficios de ley según correspondía. } & $62,50 \%$ & $37,50 \%$ \\
\hline 2 & \multicolumn{2}{|c|}{ Hubo otros compañeros en la misma empresa que perdieron sus empleos... } & $93,75 \%$ & $6,25 \%$ \\
\hline 3 & \multicolumn{2}{|c|}{ En mi familia hubo personas que perdieron sus empleos durante la pandemia. } & $81,25 \%$ & $18,75 \%$ \\
\hline 4 & \multicolumn{2}{|c|}{ La empresa que me despidió se declaró en quiebra. } & $25 \%$ & $75 \%$ \\
\hline 5 & \multicolumn{2}{|c|}{ Actualmente ya poseo otro empleo. } & $62,5 \%$ & $37,5 \%$ \\
\hline 6 & \multicolumn{2}{|c|}{ La empresa nos ofreció posibilidad de reenganche en un futuro... } & $0,00 \%$ & $100,00 \%$ \\
\hline 7 & \multicolumn{2}{|c|}{ Si no me hubiesen despedido, igualmente hubiese renunciado poco después. } & $0,00 \%$ & $100,00 \%$ \\
\hline
\end{tabular}

Fuente: elaboración propia

Finalmente, un total de 16 hombres perdieron su puesto de trabajo (32\%) siendo más evidente esto en el sector público (Tabla 5). De los despedidos, la mayoría indicó que, si le pagaron los 
beneficios de ley, indicaron que familiares y amigos habían perdido también sus puestos de trabajo. Por otro lado, no les ofrecieron la posibilidad de reenganche de futuro y no tenían, previo a su despido, intenciones de abandonar su trabajo.

Según los estudios realizados por la Revista digital Primicias (Coba G. , 2020) se menciona que, en junio de 2020, la tasa de desempleo en Quito fue de 22,8\%, es decir, 7,9 puntos porcentuales por encima de la tasa de Guayaquil y 9,5 puntos porcentuales sobre la tasa de desempleo nacional.

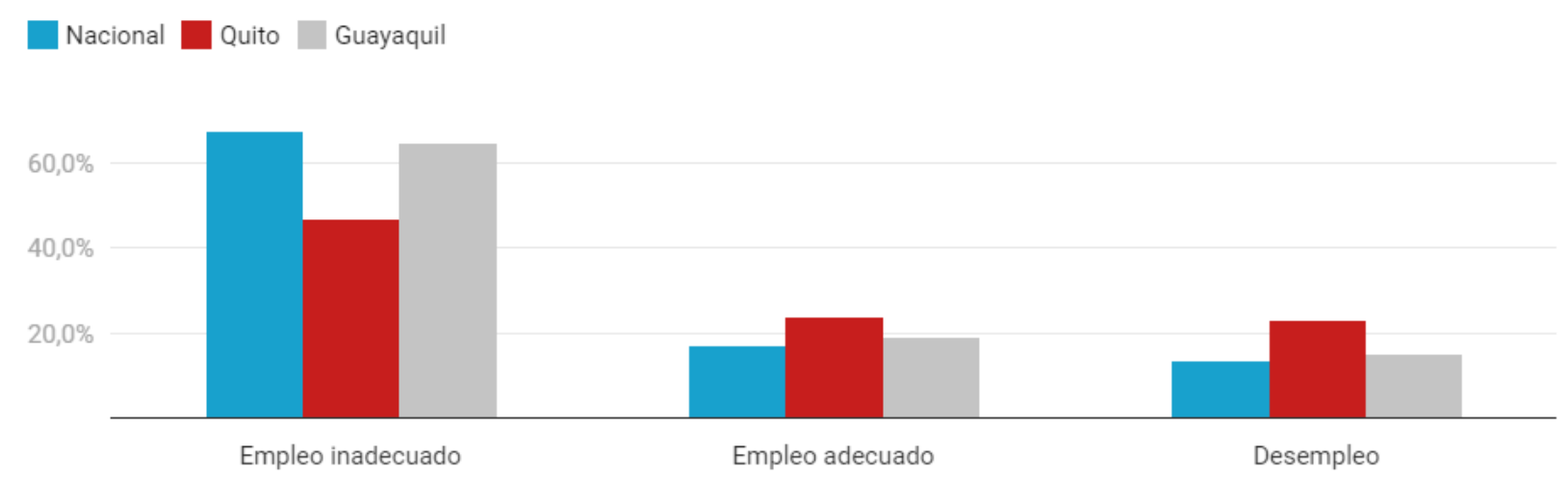

Figura 1. Evolución del empleo mayo/junio 2020

Fuente: Cámara de Comercio de Quito

En la Figura 1 podemos observar que el $40 \%$ de empleo inadecuado se encuentra en la ciudad de Quito, así como el $20 \%$ de empleo adecuado y finalmente una tasa de desempleo del $20 \%$, esto en los meses de mayo/junio de 2020.

De la misma manera, en el mes de mayo, El Observatorio de la PyME de la Universidad Andina Simón Bolívar, sede Ecuador, preocupado por ir generando información sobre lo que viene sucediendo en el país alrededor de la pandemia (covid-19), decidió llevar a cabo el estudio Panorama Actual y Perspectivas Futuras sobre los efectos del COVID-19 en el empleo y la Economía del Ecuador, el cual tuvo como objetivo central conocer las percepciones sobre los efectos que, la pandemia que vive el Ecuador y el mundo en general, ha ocasionado en el comportamiento del empleo y las expectativas futuras que van emergiendo sobre la evolución de la economía ecuatoriana (Jaramillo, 2020), obteniendo así el siguiente resultado acerca de la percepción de las condiciones laborales. 


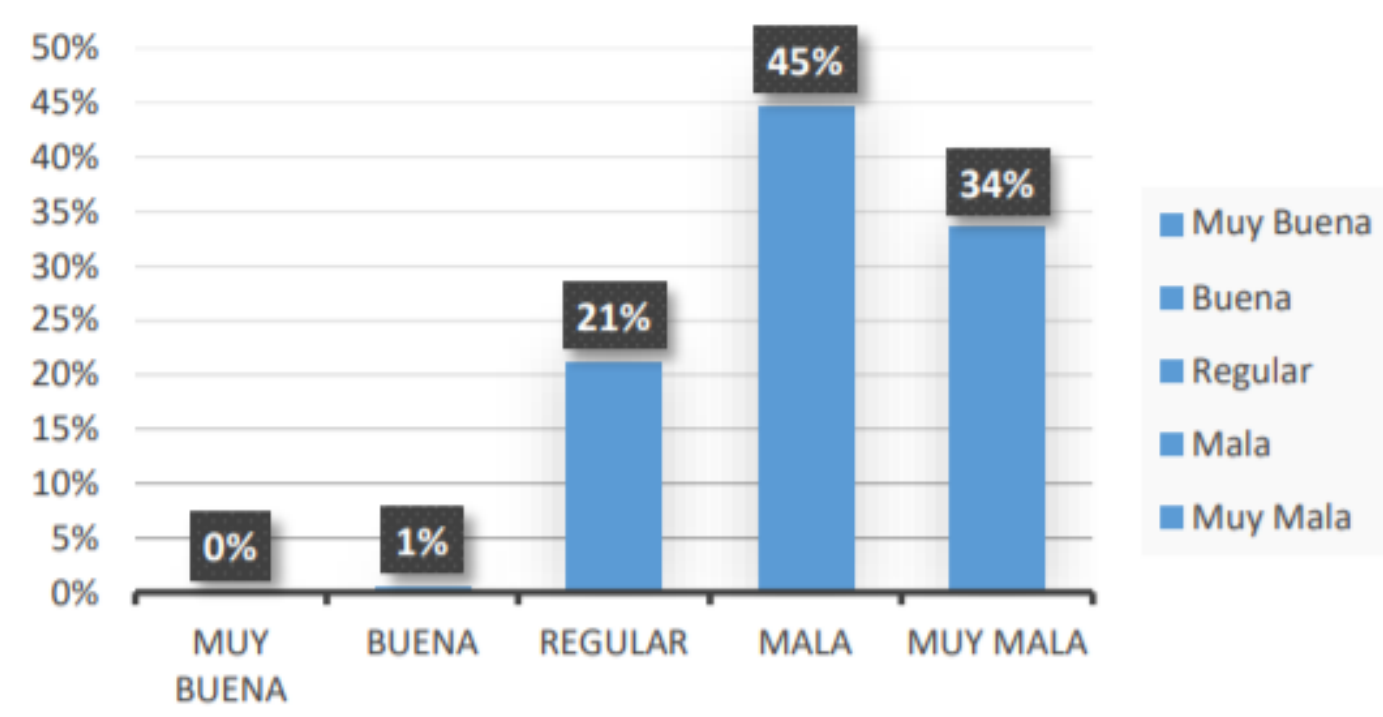

Figura 2. Percepción sobre la situación laboral actual del país

Fuente: Universidad Andina Simón Bolívar - Sede Ecuador

En la Figura 2 se puede observar que la percepción de la mayoría de personas económicamente activas se encuentra insatisfechos con las condiciones laborales, calificándolas así, como mala y muy mala, es importante recalcar que este estudio se realizó a una muestra de 349 personas, de las cuales el $80 \%$ son de la ciudad de Quito.

Es importante mencionar que la aparición de la pandemia también trajo consigo aspectos positivos en el sector laboral, las empresas se vieron en la necesidad de adaptarse a una nueva modalidad mejorando su infraestructura tecnológica para facilitar la conexión remota de sus colaboradores. Otras organizaciones optaron por las ventas online, implementado plataformas digitales que le permitieron comercializar sus productos y mantener sus ingresos, ayudando así a la economía del cantón.

El desempleo permitió que muchos identificaran oportunidades de un nuevo emprendimiento que les permita obtener una estabilidad económica.

Según Enriquez (2020), el 82,7\% de ecuatorianos emprende por falta de empleo, 52,7\% por marcar una diferencia; $36,5 \%$ por acumular riqueza y el 35,7 \% por continuar una tradición familiar.

Según cifras de la Superintendencia de Compañías (citado en El Universo, 2020), de enero al 8 de mayo del 2020 se constituyeron en el país 1856 nuevas empresas, el 43,96\%. Finalmente, es relevante mencionar que el 22 de junio de 202 se publicó en el Registro Oficial la Ley Orgánica de Apoyo Humanitario para combatir la crisis sanitaria derivada del Covid-19, en la cual se otorgaba facultades a los empleados para reducción de jornadas lo cual conlleva una reducción salarial, ajustada a las horas laboradas; esto como los principales puntos que llevaron a inconformidades por parte de los colaboradores tanto de la empresa pública como privada. 


\section{Conclusiones}

De acuerdo a lo desarrollado se concluye que el impacto del COVID-19 en el empleo de la ciudad de Quito responde a tres dimensiones básicas.

- Pérdida significativa de empleos que afectan principalmente a las mujeres y una parcial afectación a las condiciones de empleo pleno que ha provocado una sobrecarga laboral y desmejora de los beneficios percibidos por los trabajadores.

- Como producto del desempleo muchos quiteños decidieron prepararse e iniciar con un emprendimiento, lo cual no solo ayudó a la economía de su hogar, sino también a la del cantón.

- Las posibilidades de mejora para las condiciones laborales de los trabajadores, se puede llevar a cabo con diversas actividades que busquen disminuir el estrés y sobrecarga laboral que tenemos como resultado de la nueva modalidad laboral y éstas serán responsabilidad de cada organización, considerando su giro de negocio. 


\section{Referencias}

Arce, O. (2020). El impacto de la crisis del COVID-19 sobre el empleo de los jóvenes y las mujeres. Informe El trabajador del futuro: Conectado, Versátil y Seguro. https://www.bde.es/f/webbde/GAP/Secciones/SalaPrensa/IntervencionesPublicas/DirectoresGenerales/economia/Arc/arce080720.pdf

Banco Central del Ecuador. (30 de septiembre de 2020). La economía ecuatoriana decreció 12,4\% en el segundo trimestre de 2020. Boletines de Prensa. Quito, Pichincha, Ecuador. Obtenido de https://www.bce. fin.ec/index.php/boletines-de-prensa-archivo/item/1383-la-economia-ecuatoriana-decrecio-12-4-en-el-segundo-trimestre-de-2020

Cevallos, G., Calle, A., \& Ponce, O. (2020). Impacto social causado por la COVID-19 en Ecuador. 3C Empresa. Investigación y pensamiento crítico, 44(2), 115-127. https://doi.org/10.17993/3cemp.2020.edicionespecial1.115-127

Coba, G. (16 de septiembre de 2020). Quito: tres indicadores muestran impacto de la pandemia en la economía. https://www.primicias.ec/noticias/economia/indicadores-quito-ciudad-golpeada-crisis-covid/

Delgado Jurado, G. I. (2020). Análisis de la Ley Orgánica de Apoyo Humanitario para enfrentar la crisis de la pandemia del COVID 19 dirigido al empleo público y privado. Escuela de Posgrado "ESPOG". Universidad Israel. Obtenido de http://repositorio.uisrael.edu.ec/bitstream/47000/2691/1/UISRAEL-EC-MASTERADMP-378.242-2020-046.pdf

El Universo. (18 de mayo de 2020). La creación de empresas cae un 43,96 \% en Ecuador en medio de la emergencia por el COVID-19. https://www.eluniverso.com/noticias/2020/05/17/nota/7843821/constitucion-empresas-notarias-covid-19/

Enriquez, C. (27 de julio de 2020). Cinco aspectos positivos y negativos sobre la realidad de los lugares de trabajo poscovid-19. Revista líderes. https://www.revistalideres.ec/lideres/oficinas-coronavirus-teletrabajo-pandemia-encuesta.html

González, M. J. (2017). Políticas activas de empleo en la UE en tiempos de crisis económica. Universidad Complutense de Madrid, Departamento de Economía Aplicada III, Madrid. https://eprints.ucm.es/40750/

Jaramillo, W. A. (18 de mayo de 2020). Estudio sobre los efectos del COVID 19 en el empleo y la Economía del Ecuador. Universidad Andina Simón Bolívar, Sede Ecuador. https://www.uasb.edu.ec/web/observatorio-pyme/contenido?estudio-sobre-los-efectos-del-covid-19-en-el-empleo-y-la-economia-del-ecuador

Kubicek, B., \& Korunka, C. (2017). Job demands in a changing world of work. En Research Handbook on Work and Well-Being (págs. 59-76). Editorial Elgar. https://doi.org/10.4337/9781785363269.00009 
López Roldán, P., \& Fachelli, S. (2015). Metodología de la Investigación Social Cuantitativa. Barcelona, España: Universidad Autónoma de Barcelona. https://ddd.uab.cat/pub/caplli/2016/163565/metinvsoccua a2016 cap1-3.pdf

Martínez, C. A. (2017). Cuatro niveles de conocimiento en relación a la ciencia. Una propuesta taxonómica. Ciencia Ergo-sum, 24(1), 83-90. https://doi.org/10.30878/ces.v24n1a9

Quevedo Barros, M. R., Vásquez Lafebre, L. M., Quevedo Vasquez, J. O., \& Pinzón Prado, L. T. (2020). COVID-19 y sus efectos en el comercio internacional. Caso Ecuador. Dominio de las Ciencias, 6(3). https:// doi.org/10.23857/POCAIP

Sampieri, R. (2003). Metodología de la Investigación. México: McGraw-Hill Interamericana.

Young Lee, J., \& Lee, Y. (25 de julio de 2018). Job Crafting and Performance: Literature Review and Implications for Human Resource Development. Human Resource Development Review, 17(3), 277-313. https://doi. org/10.1177/1534484318788269 
Copyright (c) 2021 Michel Esthefanía Arévalo Ávila, Jhonnatan Vinicio Reascos Tapia y Héctor Sebastián Pérez Manosalvas

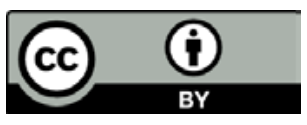

Este texto está protegido bajo una licencia internacional Creative Commons 4.0.

Usted es libre para Compartir-copiar y redistribuir el material en cualquier medio o formato - y Adaptar el documento - remezclar, transformar y crear a partir del material-para cualquier propósito, incluso para fines comerciales, siempre que cumpla las condiciones de Atribución. Usted debe dar crédito a la obra original de manera adecuada, proporcionar un enlace a la licencia, e indicar si se han realizado cambios. Puede hacerlo en cualquier forma razonable, pero no de forma tal que sugiera que tiene el apoyo del licenciante o lo recibe por el uso que hace de la obra.

\section{$\underline{\text { Resumen de licencia }-\underline{T e x t o ~ c o m p l e t o ~ d e ~ l a ~ l i c e n c i a ~}}$}

